\title{
SYSTEMS OF ORTHOGONAL POLYNOMIALS ON CERTAIN ALGEBRAIC CURVES ${ }^{1}$
}

\author{
FULTON KOEHLER
}

1. Introduction. A system of orthogonal polynomials in two real variables $x$ and $y$ can be defined with respect to any domain of integration in the $x y$ plane and any nonnegative weight function which has a positive integral over that domain. Although a general study of the formal properties of such systems has been made, ${ }^{2}$ the corresponding problem of convergence has been adequately dealt with only for cases which reduce almost immediately to familiar problems in a single variable. The purpose of this paper is to give more substantial examples of systems for which it is possible, by special methods, to present an account of convergence which is fully comparable, at least in some of its main features, with such highly developed theories as those of Fourier and Legendre series.

The proofs, as in the case of series of orthogonal polynomials in one variable with a more or less general weight function, can be made to depend on properties of boundedness. The method used here for obtaining these properties is to establish relationships between the systems in two variables considered and systems in one variable whose properties are well known.

The domain of integration to which primary consideration is to be given is the perimeter of the square whose sides are segments of the lines $x= \pm 1, y= \pm 1$. Two other domains will be dealt with briefly in a concluding section.

The square contour will be denoted by the letter $C$. We shall take the following sequence as the basis for the construction of a set of orthogonal polynomials on $C: 1, x+y, x-y, x y, x^{2}+y^{2}, x^{2}-y^{2}$, $x^{2} y+x y^{2}, x^{2} y-x y^{2}, x^{3}+y^{3}, x^{3}-y^{3}, \cdots, x^{n-1} y+x y^{n-1}, x^{n-1} y-x y^{n-1}$, $x^{n}+y^{n}, x^{n}-y^{n}, \cdots$. The terms of this sequence have the property that any finite number of them are linearly independent on $C$; and also, by means of the identity $x^{2} y^{2}-x^{2}-y^{2}+1 \equiv 0$ which holds everywhere on $C$, any polynomial in $x$ and $y$ can be expressed on $C$ as a linear combination of them. If $\rho(x, y)$ is a function which is positive almost everywhere on $C$, we can multiply each member of the sequence by $\rho^{1 / 2}$ and apply the Schmidt process of orthogonalization,

${ }^{1}$ Presented to the Society, April 14, 1939.

2 D. Jackson, (1) Formal properties of orthogonal polynomials in two variables, Duke Mathematical Journal, vol. 2 (1936), pp. 423-434. (2) Orthogonal polynomials on a plane curve, Duke Mathematical Journal, vol. 3 (1937), pp. 228-236. 
thus obtaining an orthogonal system of polynomials with respect to the weight function $\rho(x, y)$. Let these polynomials be normalized and given positive leading coefficients, where the leading coefficient of a polynomial is defined as being the coefficient of the last member of the above sequence which appears in it. We shall denote the polynomials thus uniquely defined by $p_{n k}(x, y)$, where $n$ is the degree of the polynomial, and $k$ is $1,2,3$, or 4 according as the leading term is the first, second, third, or fourth term of the $n$th degree in the above sequence.

2. Boundedness of the normalized polynomials for some weight functions. Let $P_{n}[x ; f(x)]$ be the polynomial of the $n$th degree in the normalized orthogonal system on the interval $(-1,1)$ corresponding to the weight function $f(x)$.

Theorem I. If $\rho(x, y)=\rho(y, x)=\rho(-x,-y)$ for all points $(x, y)$ of $C$, and if the polynomials $P_{n}[x ; \rho(x, 1)]$ are uniformly bounded for all values of $n$ on every closed interval interior to $(-1,1)$, then the polynomials $p_{n k}(x, y)$ are uniformly bounded for all values of $n$ and $k$ on every closed point set of $C$ not including one of the corner points.

To prove the theorem it is first necessary to point out certain facts regarding integration over the contour $C$. The integral of any function $g(x, y)$ over $C$ is given by the formula

$$
\begin{aligned}
\int_{C} g(x, y) d s= & \int_{-1}^{1} g(x, 1) d x+\int_{-1}^{1} g(x,-1) d x \\
& +\int_{-1}^{1} g(1, y) d y+\int_{-1}^{1} g(-1, y) d y .
\end{aligned}
$$

Hence, if $g(x, y)=-g(y, x)$ at all points of $C$ or if $g(x, y)=-g(-x,-y)$ at all points of $C, \int_{C} g(x, y) d s=0$. From this it is possible to prove that each polynomial $p_{n k}(x, y)$ is either symmetric or skew-symmetric and that each is either even or odd; that is, $p_{n k}(x, y) \equiv \pm p_{n k}(y, x)$ and $p_{n k}(x, y) \equiv \pm p_{n k}(-x,-y)$.

The polynomial $p_{2 n, 1}(x, y)$ is therefore a linear combination of the terms $x^{2 n-1} y+x y^{2 n-1}, x^{2 n-2}+y^{2 n-2}, x^{2 n-3} y+x y^{2 n-3}, \cdots, x^{2}+y^{2}, x y, 1$. It has the following properties of orthogonality:

$$
\begin{aligned}
\int_{C} \rho(x, y) p_{2 n, 1}(x, y)\left(x^{2 k}+y^{2 k}\right) d s & =0, \quad k=0,1, \cdots, n-1, \\
\int_{C} \rho(x, y) p_{2 n, 1}(x, y)\left(x^{2 k-1} y+x y^{2 k-1}\right) d s & =0, \quad k=1,2, \cdots, n-1 .
\end{aligned}
$$


The integrands in these two equations are even and symmetric and the integral over $C$ of such a function is equal to four times the integral of that function over any one side of $C$. Hence,

$$
\begin{aligned}
\int_{-1}^{1} \rho(x, 1) p_{2 n, 1}(x, 1)\left(x^{2 k}+1\right) d x=0, & k=0,1, \cdots, n-1, \\
\int_{-1}^{1} \rho(x, 1) p_{2 n, 1}(x, 1)\left(x^{2 k-1}+x\right) d x & =0, \quad k=1,2, \cdots, n-1 .
\end{aligned}
$$

That is, $p_{2 n, 1}(x, 1)$ is a polynomial in $x$ of degree $2 n-1$ which is orthogonal over $(-1,1)$ with $\rho(x, 1)$ as weight function to $1, x, x^{2}, \cdots$, $x^{2 n-2}$. Hence,

$$
p_{2 n, 1}(x, 1)=(1 / 2) P_{2 n-1}[x ; \rho(x, 1)] .
$$

By similar methods it can be shown that $p_{2 n, 2}(x, 1) /\left(x^{2}-1\right)$ is a polynomial of degree $2 n-3$ which is orthogonal over $(-1,1)$ with $\left(1-x^{2}\right)^{2} \rho(x, 1)$ as weight function to $1, x, x^{2}, \cdots, x^{2 n-4}$; and so

$$
p_{2 n, 2}(x, 1)=(1 / 2)\left(x^{2}-1\right) P_{2 n-3}\left[x ;\left(1-x^{2}\right)^{2} \rho(x, 1)\right] .
$$

The corresponding formulas for the other two polynomials of even degree and the four of odd degree are

$$
\begin{aligned}
p_{2 n, 3}(x, 1) & =(1 / 2) P_{2 n}[x ; \rho(x, 1)], \\
p_{2 n, 4}(x, 1) & =(1 / 2)\left(x^{2}-1\right) P_{2 n-2}\left[x ;\left(1-x^{2}\right)^{2} \rho(x, 1)\right], \\
p_{2 n+1,1}(x, 1) & =(1 / 2)(x+1) P_{2 n-1}\left[x ;(1+x)^{2} \rho(x, 1)\right], \\
p_{2 n+1,2}(x, 1) & =(1 / 2)(x-1) P_{2 n-1}\left[x ;(1-x)^{2} \rho(x, 1)\right], \\
p_{2 n+1,3}(x, 1) & =(1 / 2)(x+1) P_{2 n}\left[x ;(1+x)^{2} \rho(x, 1)\right], \\
p_{2 n+1,4}(x, 1) & =(1 / 2)(x-1) P_{2 n}\left[x ;(1-x)^{2} \rho(x, 1)\right] .
\end{aligned}
$$

It is well known that if the polynomials $P_{n}[x ; f(x)]$ are uniformly bounded on every closed interval interior to $(-1,1)$, then the polynomials $P_{n}[x ; \pi(x) f(x)]$ are likewise bounded provided $\pi(x)$ is a polynomial which does not vanish on the interior of the interval $(-1,1)$. Hence, the above formulas, together with the fact that each polynomial $p_{n k}(x, y)$ is symmetric or skew-symmetric and even or odd, establish the theorem.

It is of interest to note that if $\rho(x, y)=|y+x|^{\alpha}|y-x|^{\beta}$ where $\alpha>-1, \beta>-1$, then the polynomials $p_{n k}(x, 1)$ are expressible in terms of Jacobi polynomials.

3. Convergence theorems. Let $f(x, y)$ be any function of $x$ and $y$ 
which is integrable in the sense of Lebesgue over $C$ and let us consider the series

$$
\sum_{n=0}^{\infty} \sum_{k=1}^{4} a_{n k} p_{n k}(x, y)
$$

where $a_{n k}=\int_{C} \rho(u, v) f(u, v) p_{n k}(u, v) d s$ and where $(x, y)$ is any point of $C$. (For convenience of notation we define $p_{02}, p_{03}, p_{04}, p_{13}, p_{14}$, and $p_{24}$ to be identically zero.) Let $K_{n}(x, y, u, v)=\sum_{m=0}^{n} \sum_{k=1}^{4} p_{m k}(x, y) p_{m k}(u, v)$, and let $S_{n}(x, y)$ be the partial sum of (1) through terms of the $n$th degree. Then

$$
S_{n}(x, y)-f(x, y)=\int_{C} \rho(u, v)[f(u, v)-f(x, y)] K_{n}(x, y, u, v) d s .
$$

(In formulas where $x, y, u$, and $v$ appear under the integral sign $u$ and $v$ are to be taken as the variables of integration.) By using a formula ${ }^{3}$ for $K_{n}(x, y, u, v)$ analogous to the Christoffel-Darboux identity, the difference $S_{n}(x, y)-f(x, y)$ can be expressed as a sum of at most thirty-two terms of the form

$$
k p_{j h}(x, y) \int_{C} \rho(u, v) \frac{f(u, v)-f(x, y)}{(A u+B v)-(A x+B y)} p_{m k}(u, v) d s
$$

where $A$ and $B$ are any arbitrary constants, where $k$ is a constant numerically less that $|A|+|B|$, and where $m$ and $j$ take on the values $n$ and $n+1$. The problem of convergence at a specified point thus reduces to proving that an expression of the form (3) approaches zero as $n$ becomes infinite.

LEMMA 1. If the weight function $\rho(x, y)$ is of such a nature that the corresponding polynomials are uniformly bounded on every closed point set of $C$ not containing a corner point and if $\phi(x, y)$ is a function such that $\rho \phi$ is integrable over $C$ and $\rho \phi^{2}$ is integrable over some neighborhood on $C$ of each corner point, then

$$
\lim _{n \rightarrow \infty} \int_{C} \rho(u, v) \phi(u, v) p_{n k}(u, v) d s=0, \quad k=1,2,3,4 .
$$

The proof of this lemma is omitted since it can be easily constructed from proofs already known. ${ }^{4}$ On the basis of this lemma we can now prove the following assertion:

${ }^{3}$ D. Jackson, (1), p. 433.

${ }^{4}$ See, for example, D. Jackson, Series of orthogonal polynomials, Annals of Mathematics, (2), vol. 34 (1933), pp. 536-537. 
Theorem II. Assume

(a) the weight function $\rho(x, y)$ is such that the corresponding polynomials $p_{n k}(x, y)$ are uniformly bounded on every closed point set of $C$ not containing a corner point;

(b) $(x, y)$ is a point of $C$, not a corner;

(c) the function of is integrable over $C$ and $\rho f^{2}$ is integrable over a neighborhood on $C$ of each corner point;

(d) the function

$$
\rho(u, v) \frac{f(u, v)-f(x, y)}{(u-x)+(v-y)}
$$

is integrable over some neighborhood on $C$ of the point $(x, y)$.

Then the series (1) converges at the point $(x, y)$ to the value $f(x, y)$.

Let us first make the additional hypothesis that there is a point $\left(x^{\prime}, y^{\prime}\right)$ on $C$ on the interior of a side different from the one containing $(x, y)$ such that the function

$$
\rho(u, v) \frac{f(u, v)-f(x, y)}{\left(u-x^{\prime}\right)+\left(v-y^{\prime}\right)}
$$

is integrable over some neighborhood on $C$ of the point $\left(x^{\prime}, y^{\prime}\right)$. Determine $A$ and $B$ so that the straight line $A(u-x)+B(v-y)=0$ in the $u v$ plane passes through the point $\left(x^{\prime}, y^{\prime}\right)$. Then in some neighborhood on $C$ of $(x, y)$ the expression $A(u-x)+B(v-y)$ is a nonvanishing constant multiple of $u-x+v-y$ and in some neighborhood of $\left(x^{\prime}, y^{\prime}\right)$, of $u-x^{\prime}+v-y^{\prime}$. If we let

$$
\phi(u, v)=\frac{f(u, v)-f(x, y)}{A(u-x)+B(v-y)},
$$

then $\rho \phi$ is integrable over $C$ and $\rho \phi^{2}$ is integrable over a neighborhood of each corner point; hence, by Lemma 1, the entire expression (3) approaches zero as $n$ becomes infinite, which insures the convergence of $(1)$ to the value $f(x, y)$.

To prove the theorem in the general case let us choose two distinct points $\left(x^{\prime}, y^{\prime}\right)$ and $\left(x^{\prime \prime}, y^{\prime \prime}\right)$, each on the interior of a side of $C$ different from the side containing $(x, y)$, and let us define a function $g(u, v)$ to be equal to $f(u, v)$ throughout neighborhoods of $(x, y)$ and $\left(x^{\prime}, y^{\prime}\right)$, equal to $g(x, y)=f(x, y)$ throughout a neighborhood of $\left(x^{\prime \prime}, y^{\prime \prime}\right)$, and equal to zero elsewhere on $C$. Then the series of form (1) corresponding to the functions $f-g$ and $g$ will converge at the point $(x, y)$ to the values $f(x, y)-g(x, y)$ and $g(x, y)$ respectively. Hence, the series for $f$ will converge at the point $(x, y)$ to the value $f(x, y)$. 
COROLLARY. If the function $f$ is such that of is integrable over $C$ and $\rho f^{2}$ is integrable over a neighborhood of each corner point, the behavior of the series (1) at a point $(x, y)$ on the interior of a side of $C$ depends only on the behavior of the function $f(u, v)$ in the neighborhood of that point.

A study of the uniform convergence of (1) to a continuous function $f(x, y)$ can be made with the same methods that have been used in a study of Legendre series..$^{5}$ In applying these methods it is first necessary to consider the degree of polynomial approximation obtainable over the contour $C$ to a function which is continuous on $C$. By adaptation of existing theorems ${ }^{6}$ with regard to polynomial approximation on an interval, the following conclusion is obtained:

THEOREM III. If the polynomials $p_{n k}(x, y)$ are uniformly bounded and the weight function $\rho(x, y)$ bounded on every closed point set of $C$ not including one of the corner points and if $f(x, y)$ is a function such that $f(x, \pm 1)$ and $f( \pm 1, x)$ have continuous pth derivatives $(p \geqq 0)$ on the interval $(-1,1)$ with moduli of continuity at most equal to $\omega(\delta)$; then, for every $\eta>0$,

$$
\left|S_{n}(x, y)-f(x, y)\right| \leqq \frac{K \log n}{n^{p}} \omega(1 / n)
$$

for all $(x, y)$ on $C$ not within a distance $\eta$ of any corner point and for all $n>1$, the constant $K$ being independent of $n$ and of $(x, y)$ but dependent on $\eta$.

4. Other contours. The methods used in the convergence proofs of the preceding section are applicable to orthogonal systems over a highly general contour provided the normalized polynomials of the system possess the requisite property of boundedness on that contour. There has been as yet, however, no general method devised for establishing the latter property. Two other ranges of integration for which the result can be established by reasoning similar to that already used are a pair of bisecting line segments and a pair of concentric circles.

An orthogonal system over the first of these domains will have two polynomials of the $n$th degree for $n \geqq 1$. If the range is taken as consisting of the portions of the $x$ and $y$ axes contained in the intervals $-1 \leqq x \leqq 1,-1 \leqq y \leqq 1$, the two polynomials of $n$th degree of a normalized, orthogonal system corresponding to a symmetric weight

\footnotetext{
5 See, for example, D. Jackson, The Theory of Approximation, American Mathematical Society Colloquium Publications, vol. 11, New York, 1930, pp. 25-32.

${ }^{6}$ D. Jackson, loc. cit., pp. 13-18.
} 
function $\rho(x, y)$ are given by the formulas

$$
\begin{gathered}
p_{n 1}(x, 0)=2^{-1 / 2} P_{n}[x ; \rho(x, 0)], \quad p_{n 2}(x, 0)=2^{-1 / 2} x P_{n-1}\left[x ; x^{2} \rho(x, 0)\right], \\
p_{n k}(x, y)=(-1)^{k-1} p_{n k}(y, x) .
\end{gathered}
$$

The convergence theorems for this system are similar to those of the last section with the points $( \pm 1,0),(0, \pm 1),(0,0)$ appearing as exceptional points analogous to the corner points of the square.

The contour made up of two concentric circles is an example of a contour which has no singular points. An orthogonal system over this contour will have four polynomials of the $n$th degree for $n \geqq 3$. If the two circles are taken with center at the origin and radii $a$ and $b$, the polynomials of a normalized system corresponding to the weight function 1 are represented by the formulas

$$
\begin{aligned}
& p_{n k}(a \cos \theta, a \sin \theta)=\alpha_{n k 1} \cos n \theta+\alpha_{n k 2} \cos (n-2) \theta, \\
& p_{n k}(b \cos \theta, b \sin \theta)=\beta_{n k 1} \cos n \theta+\beta_{n k 2} \cos (n-2) \theta, \quad k=1,3 .
\end{aligned}
$$

The formulas for $p_{n 2}$ and $p_{n 4}$ are of the same form with the cosines replaced by sines. The constants $\alpha_{n k m}$ and $\beta_{n k m}$ involved in the formulas are bounded for all values of $n, k$, and $m$; so the polynomials themselves are uniformly bounded over the entire contour. The convergence theorems for this system of polynomials can thus be formulated without reference to any exceptional points.

The University of Minnesota 